\title{
A short proof of the deformation property of Bridgeland stability conditions
}

\author{
Arend Bayer $^{1}$ (D) \\ Received: 13 July 2018 / Revised: 20 August 2019 / Published online: 10 September 2019 \\ (C) The Author(s) 2019
}

\begin{abstract}
The key result in the theory of Bridgeland stability conditions is the property that they form a complex manifold. This comes from the fact that given any small deformation of the central charge, there is a unique way to correspondingly deform the stability condition. We give a short direct proof of an effective version of this deformation property.
\end{abstract}

\section{Contents}

1 Introduction . . . . . . . . . . . . . . . . . . . . . . . . . . 1597

2 Review: definitions and basic properties . . . . . . . . . . . . . . . . . . . . . . . 1599

3 Harder-Narasimhan filtrations via the Harder-Narasimhan polygon . . . . . . . . . . . . . . . 1602

4 Linear algebra lemmas . . . . . . . . . . . . . . . . . . . . . . . . . . . . . . . . . . . 1604

5 Real variations of the central charge . . . . . . . . . . . . . . . . . . . . . . . 1606

6 The support property is preserved . . . . . . . . . . . . . . . . . . . . . . . 1608

7 Reductions ..................................... 1610

8 Application ............................... 1611

References . . . . . . . . . . . . . . . . . . . . . . . 1612

\section{Introduction}

Stability conditions on triangulated categories, introduced in [9], have been hugely influential, due to their connections to physics [13,14], to mirror symmetry [11] and to representation theory [2], and due to their applications within algebraic geometry, for

Communicated by Vasudevan Srinivas.

Arend Bayer

arend.bayer@ed.ac.uk

http://www.maths.ed.ac.uk/ abayer/

1 School of Mathematics and Maxwell Institute, University of Edinburgh, James Clerk Maxwell Building, Peter Guthrie Tait Road, Edinburgh EH9 3FD, UK 
example to Donaldson-Thomas invariants [23], to the derived category itself $[4,15]$, or to the birational geometry of moduli spaces $[1,3,6,18,20]$.

Their distinguishing property, crucial in all applications, is a strong deformation property: by [9, Theorem 1.2], there is a complex manifold of stability conditions, with a map to a vector space that is a local isomorphism. We give a short proof of an effective version of this result.

Result. We refer to Sect. 2 for complete definitions; here we review notation and the support property. Let $\mathcal{D}$ be a triangulated category, and let $v: K(\mathcal{D}) \rightarrow \Lambda$ be a homomorphism from its K-group to a finitely generated free abelian group $\Lambda$. A pre-stability condition on $\mathcal{D}$ with respect to $v$ is a pair $\sigma=(Z, \mathcal{P})$, where $\mathcal{P}$ is a slicing (see Definition 2.1) and $Z: \Lambda \rightarrow \mathbb{C}$ is a compatible (see Definition 2.2) group homomorphism.

Definition 1.1 $[9,17]$ Let $Q: \Lambda_{\mathbb{R}} \rightarrow \mathbb{R}$ be a quadratic form. We say that a pre-stability condition $(Z, \mathcal{P})$ satisfies the support property with respect to $Q$ if

1. the kernel $\operatorname{Ker} Z \subset \Lambda_{\mathbb{R}}$ of the central charge is negative definite with respect to $Q$, and

2. for any semistable object $E$, i.e. $E \in \mathcal{P}(\phi)$ for some $\phi \in \mathbb{R}$, we have $Q(v(E)) \geq 0$.

In this case, we call $\sigma$ a stability condition. Let $\operatorname{Stab}_{\Lambda}(\mathcal{D})$ denote the topological space (see Sect. 2) of stability conditions, and $\mathcal{Z}: \operatorname{Stab}_{\Lambda}(\mathcal{D}) \rightarrow \operatorname{Hom}(\Lambda, \mathbb{C})$ the map $\mathcal{Z}(Z, \mathcal{P})=Z$.

Theorem 1.2 Let $Q$ be a quadratic form on $\Lambda \otimes \mathbb{R}$, and assume that the stability condition $\sigma=(Z, \mathcal{P})$ satisfies the support property with respect to $Q$. Then:

1. There is an open neighbourhood $\sigma \in U_{\sigma} \subset \operatorname{Stab}_{\Lambda}(\mathcal{D})$ such that the restriction $\mathcal{Z}: U_{\sigma} \rightarrow \operatorname{Hom}(\Lambda, \mathbb{C})$ is a covering of the set of $Z^{\prime}$ such that $Q$ is negative definite on $\operatorname{Ker} Z^{\prime}$.

2. All stability conditions in $U_{\sigma}$ satisfy the support property with respect to $Q$.

In other words, $\operatorname{Stab}_{\Lambda}(\mathcal{D})$ is a manifold, and any path $Z_{t} \in \operatorname{Hom}(\Lambda, \mathbb{C})$ for $t \in[0,1]$ with $Z_{0}=Z$ and $\operatorname{Ker} Z_{t}$ negative definite for all $t \in[0,1]$ lifts uniquely to a continuous path $\sigma_{t}=\left(Z_{t}, \mathcal{P}_{t}\right)$ in the space of stability conditions starting at $\sigma_{0}=\sigma$.

Part 1. is an effective variant of [9, Theorem 1.2] (which says that there is some neighbourhood of $Z_{0}$ in which paths can be lifted uniquely). The entire result first appeared as [7, Proposition A.5] with an indirect proof based on reduction to [9, Theorem 1.2].

Remarks. The support property can be a deep and interesting property in itself: a quadratic Bogomolov-Gieseker type inequality for Chern classes of semistable objects which, by Theorem 1.2, is preserved under wall-crossing.

Theorem 1.2 was crucial in [7] in order to describe an entire component of the space of stability conditions on abelian threefolds, and on some Calabi-Yau threefolds. It also greatly simplifies the construction of stability conditions on surfaces (or of tiltstability on higher-dimensional varieties [8]). In this case, the quadratic form $Q$ is the classical Bogomolov-Gieseker inequality, and Theorem 1.2 gives an open subset of 
stability conditions that otherwise has to be glued together from many small pieces (see e.g. [5, Section 4]).

Theorem 1.2 of [9] also allows for components of the space of stability conditions modelled on a linear subspace $L \subset \operatorname{Hom}(\Lambda, \mathbb{C})$. When $L$ is defined over $\mathbb{Q}$, we can recover that statement by replacing $\Lambda$ with $\Lambda / \operatorname{Ker} L$ (see [19] for examples where this is not satisfied; however, to achieve well-behaved wall-crossing one has to assume that $L$ is defined over $\mathbb{Q}$ ).

Proof idea. Our proof is based on two ideas. First, we reduce to the case where the imaginary part of $Z$ is constant; then we only have to deform stability in a fixed abelian category. Secondly, we use the elementary convex geometry of the HarderNarasimhan polygon, see Sect. 3.

This avoids the need for quasi-abelian categories, and some of the more technical arguments of [9, Section 7]. We still need a few arguments similar to ones in [9], which we have reproduced for the convenience of the reader.

Application. Assume that $\mathcal{D}$ is a 2-Calabi-Yau category, i.e. for all $E, F \in \mathcal{D}$ we have a bi-functorial isomorphism $\operatorname{Hom}(E, F)=\operatorname{Hom}(F, E[2])^{\vee}$. Let $\Lambda$ be the numerical $K$-group of $\mathcal{D}$, and assume that $\Lambda$ is finitely generated. Then there is a surjection $v: K(\mathcal{D}) \rightarrow \Lambda$, and $\Lambda$ admits a non-degenerate bilinear form (_, _ ), called Mukaipairing, with

$$
\chi(E, F)=-(v(E), v(F)) .
$$

Let $\mathcal{P}_{0}(\mathcal{D}) \subset \operatorname{Hom}(\Lambda, \mathbb{C})$ be the set of central charges $Z$ such that $\operatorname{Ker} Z$ is negative definite with respect to the Mukai pairing, and such that $\operatorname{Ker} Z$ contains no $\operatorname{root} \delta \in$ $\Lambda,(\delta, \delta)=-2$.

Corollary 1.3 The restriction $\mathcal{Z}^{-1}\left(\mathcal{P}_{0}(\mathcal{D})\right) \stackrel{\mathcal{Z}}{\rightarrow} \mathcal{P}_{0}(\mathcal{D})$ is a covering map.

The proof, given in Sect. 8, is fairly similar to the case of K3 surfaces [10, Proposition 8.3]. The point of including it here is to show that in terms of the support property via quadratic forms, and equipped with Theorem 1.2, the proof becomes natural and short. This result was also proved previously for preprojective algebras of quivers in $[12,16,22]$. In each of these cases, there is a connected component of $\operatorname{Stab}(\mathcal{D})$ covering a connected component of $\mathcal{P}_{0}(X)$; such statements rely crucially on non-emptiness of moduli spaces of stable objects.

\section{Review: definitions and basic properties}

Throughout, $\mathcal{D}$ will be a triangulated category, equipped with a group homomorphism

$$
v: K(\mathcal{D}) \rightarrow \Lambda
$$

from its $K$-group to an abelian group $\Lambda \cong \mathbb{Z}^{m}$.

Definitions. We first recall the main definitions from [9]. 
Definition 2.1 A slicing $\mathcal{P}$ on $\mathcal{D}$ is a collection of full subcategories $\mathcal{P}(\phi)$ for all $\phi \in \mathbb{R}$ with

1. $\mathcal{P}(\phi+1)=\mathcal{P}(\phi)[1]$ for all $\phi \in \mathbb{R}$;

2. for $\phi_{1}>\phi_{2}$ and $E_{i} \in \mathcal{P}\left(\phi_{i}\right), i=1,2$, we have $\operatorname{Hom}\left(E_{1}, E_{2}\right)=0$; and

3. for any $E \in \mathcal{D}$ there is a sequence of maps $0=E_{0} \stackrel{i_{1}}{\rightarrow} E_{1} \rightarrow \ldots \stackrel{i_{m}}{\rightarrow} E_{m}=E$ and of real numbers $\phi_{1}>\phi_{2}>\cdots>\phi_{m}$ such that the cone of $i_{j}$ is in $\mathcal{P}\left(\phi_{j}\right)$ for $j=1, \ldots, m$.

The non-zero objects of $\mathcal{P}(\phi)$ are called semistable of phase $\phi$; its simple objects are called stable. The sequence of maps in 3. is called the Harder-Narasimhan (HN) filtration of $E$.

Definition 2.2 A pre-stability condition on $\mathcal{D}$ is a pair $\sigma=(Z, \mathcal{P})$ where $Z: \Lambda \rightarrow \mathbb{C}$ is a group homomorphism and $\mathcal{P}$ a slicing, such that for all $0 \neq E \in \mathcal{P}(\phi)$, we have $Z(v(E)) \in \mathbb{R}_{>0} \cdot e^{i \pi \phi}$.

We will abuse notation and write $Z(E)$ instead of $Z(v(E))$.

Basic properties. Let $\mathrm{GL}_{2}^{+}(\mathbb{R})$ denote the group of real $2 \times 2$-matrices with positive determinant. Since $\mathrm{GL}_{2}^{+}(\mathbb{R})$ acts on $S^{1}$, its universal cover $\widetilde{G L}_{2}^{+}(\mathbb{R})$ acts on the universal cover $\mathbb{R} \rightarrow S^{1}$ given explicitly by $\phi \mapsto e^{i \pi \phi}$. For $\tilde{g} \in \widetilde{G L}_{2}^{+}(\mathbb{R})$ we will write $g$ for the corresponding element of $\mathrm{GL}_{2}^{+}(\mathbb{R})$, and $\tilde{g} . \phi$ for the given action on $\mathbb{R}$.

Proposition 2.3 There is a natural action of $\widetilde{\mathrm{GL}}_{2}^{+}(\mathbb{R})$ on the set of pre-stability conditions given by $\tilde{g} .(Z, \mathcal{P})=\left(g \circ Z, \mathcal{P}^{\prime}\right)$ where $\mathcal{P}^{\prime}(\tilde{g} . \phi)=\mathcal{P}(\phi)$.

The heart of a bounded $t$-structure is a full subcategory $\mathcal{A} \subset \mathcal{D}$ such that

$$
\mathcal{S}(\phi):= \begin{cases}\mathcal{A}[\phi] & \text { if } \phi \in \mathbb{Z} \\ 0 & \text { if } \phi \notin \mathbb{Z}\end{cases}
$$

is a slicing (see [9, Lemma 3.2]). It is automatically an abelian subcategory; and stability conditions on $\mathcal{D}$ can be constructed from slope-stability in $\mathcal{A}$.

Definition 2.4 A stability function $Z$ on an abelian category $\mathcal{A}$ is a morphism $Z: K(\mathcal{A}) \rightarrow \mathbb{C}$ of abelian groups such that for all $0 \neq E \in \mathcal{A}$, the complex number $Z(E)$ is in the semiclosed upper half plane

$$
\mathbb{H}:=\left\{z \in \mathbb{C}: \Im z>0 \text { or } z \in \mathbb{R}_{<0}\right\} .
$$

For $0 \neq E \in \mathcal{A}$ we define its phase by $\phi(E):=\frac{1}{\pi} \arg Z(E) \in(0,1]$. An object $E \in \mathcal{A}$ is called $Z$-semistable if for all subobjects $A \hookrightarrow E$, we have $\phi(A) \leq \phi(E)$.

Definition 2.5 We say that a stability function $Z$ on an abelian category $\mathcal{A}$ satisfies the $H N$ property if every object $E \in \mathcal{A}$ admits a Harder-Narasimhan (HN) filtration: a sequence $0=E_{0} \hookrightarrow E_{1} \hookrightarrow E_{2} \hookrightarrow \cdots \hookrightarrow E_{m}=E$ such that $E_{i} / E_{i-1}$ is $Z$-semistable for $i=1, \ldots, m$, with

$$
\phi\left(E_{1} / E_{0}\right)>\phi\left(E_{2} / E_{1}\right)>\cdots>\phi\left(E_{m} / E_{m-1}\right) .
$$


Proposition 2.6 [9, Proposition 5.3] To give a pre-stability condition on $\mathcal{D}$ is equivalent to giving a heart $\mathcal{A}$ of a bounded $t$-structure, and a stability function $Z$ on $\mathcal{A}$ with the HN property.

Here we tacitly assume that $Z$ factors via $K(\mathcal{A})=K(\mathcal{D}) \stackrel{v}{\rightarrow} \Lambda$. Given $(Z, \mathcal{A})$, the slicing is determined by setting $\mathcal{P}(\phi)$ to be the $Z$-semistable objects $E \in \mathcal{A}$ of phase $\phi$ for $\phi \in(0,1]$. Conversely, given $(Z, \mathcal{P})$, the heart $\mathcal{A}=\mathcal{P}(0,1]$ is the smallest extension-closed subcategory of $\mathcal{D}$ containing $\mathcal{P}(\phi)$ for $\phi \in(0,1]$. More generally, $\mathcal{P}(\phi, \phi+1]$ is a heart for every $\phi \in \mathbb{R}$.

Definition 2.7 A stability condition $\sigma$ is a pre-stability condition that satisfies the support property in the sense of Definition 1.1 with respect to some quadratic form $Q$ on $\Lambda \otimes \mathbb{R}$.

Topology and local injectivity. There is a generalised metric, and thus a topology, on the set of slicings $\operatorname{Slice}(\mathcal{D})$ given as follows. Given two slicings $\mathcal{P}, \mathcal{Q}$, we write $\phi^{ \pm}(E)$ and $\psi^{ \pm}(E)$ for the largest and smallest phase in the associated $\mathrm{HN}$ filtration of an object $E$ for $\mathcal{P}$ and $\mathcal{Q}$, respectively. Then we define the distance of $\mathcal{P}$ and $\mathcal{Q}$ by

$$
d(\mathcal{P}, \mathcal{Q}):=\sup \left\{\left|\phi^{+}(E)-\psi^{+}(E)\right|,\left|\phi^{-}(E)-\psi^{-}(E)\right|: E \in \mathcal{D}\right\} \in[0,+\infty]
$$

We recall that this distance can be computed by considering $\mathcal{P}$-semistable objects alone:

Lemma $2.8\left[9\right.$, Lemma 6.1] We have $d(\mathcal{P}, \mathcal{Q})=d^{\prime}(\mathcal{P}, \mathcal{Q})$, where the latter is defined by

$$
d^{\prime}(\mathcal{P}, \mathcal{Q}):=\sup \left\{\psi^{+}(E)-\phi, \phi-\psi^{-}(E): \phi \in \mathbb{R}, 0 \neq E \in \mathcal{P}(\phi)\right\}
$$

Proof The inequality $d(\mathcal{P}, \mathcal{Q}) \geq d^{\prime}(\mathcal{P}, \mathcal{Q})$ is immediate. For the converse, consider $E \in \mathcal{D}$, and let $A_{i}$ be one of its $\mathrm{HN}$ factors with respect to $\mathcal{P}$. Then $\psi^{+}\left(A_{i}\right) \leq \phi\left(A_{i}\right)+$ $d^{\prime}(\mathcal{P}, \mathcal{Q}) \leq \phi^{+}(E)+d^{\prime}(\mathcal{P}, \mathcal{Q})$. Hence $E$ admits no non-zero maps from $\mathcal{Q}$-stable objects of phase bigger than $\phi^{+}(E)+d^{\prime}(\mathcal{P}, \mathcal{Q})$, and so $\psi^{+}(E) \leq \phi^{+}(E)+d^{\prime}(\mathcal{P}, \mathcal{Q})$. The analogous inequality $\psi^{-}(E) \geq \phi^{-}(E)-d^{\prime}(\mathcal{P}, \mathcal{Q})$ follows similarly.

Finally, for $E \in \mathcal{D}$ we have a non-zero map $A_{1} \rightarrow E$ with $A_{1} \in \mathcal{P}\left(\phi^{+}(E)\right)$. Therefore, $\psi^{+}(E) \geq \psi^{-}\left(A_{1}\right) \geq \phi^{-}\left(A_{1}\right)-d^{\prime}(\mathcal{P}, \mathcal{Q})=\phi^{+}(E)-d^{\prime}(\mathcal{P}, \mathcal{Q})$, and so $\left|\psi^{+}(E)-\phi^{+}(E)\right| \leq d^{\prime}(\mathcal{P}, \mathcal{Q})$. Combined with the same inequality for $\psi^{-}$, the claim follows.

The topology on $\operatorname{Stab}_{\Lambda}(\mathcal{D})$ is the coarsest topology such that both forgetful maps

$$
\begin{gathered}
\operatorname{Stab}_{\Lambda}(\mathcal{D}) \rightarrow \operatorname{Slice}(\mathcal{D}), \quad(Z, \mathcal{P}) \mapsto \mathcal{P} \\
\mathcal{Z}: \operatorname{Stab}_{\Lambda}(\mathcal{D}) \rightarrow \operatorname{Hom}(\Lambda, \mathbb{C}), \quad(Z, \mathcal{P}) \mapsto Z
\end{gathered}
$$

are continuous.

The following Lemma is a variant of [9, Lemma 6.4]: 
Lemma 2.9 Assume that $\sigma=(Z, \mathcal{P})$ and $\tau=(W, \mathcal{Q})$ are two pre-stability conditions such that $\sigma$ satisfies the support property with respect to $Q$, such that

$$
\frac{|W(v)-Z(v)|}{|Z(v)|}<\sin \pi \epsilon \text { for all } v \in \Lambda \text { with } Q(v) \geq 0,
$$

and such that either $(\mathcal{P}, \mathcal{Q})<\frac{1}{4}$, or that $\sigma, \tau$ have the same heart $\mathcal{P}(0,1]=\mathcal{Q}(0,1]$. Then $d(\mathcal{P}, \mathcal{Q})<\epsilon$.

Of course, (1) means in particular that the phases of $W(v)$ and $Z(v)$ differ by at $\operatorname{most} \epsilon$.

Proof We want to apply Lemma 2.8, so let us consider an object $E \in \mathcal{P}(\phi)$. In the first case, $d(\mathcal{P}, \mathcal{Q})<\frac{1}{4}$, we apply this assumption twice to see that every HN filtration factor of $E$ with respect to $\mathcal{Q}$ is contained in $\mathcal{Q}\left(\phi-\frac{1}{4}, \phi+\frac{1}{4}\right) \subset \mathcal{P}\left(\phi-\frac{1}{2}, \phi+\frac{1}{2}\right]=: \mathcal{A}$. In particular, the first $\mathrm{HN}$ filtration factor $E_{1} \rightarrow E$ of $E$ with respect to $\mathcal{Q}$ is a subobject of $E$ in the abelian category $A$. The analogous claim is obvious in the second case for $\mathcal{A}=\mathcal{P}(0,1]$. Therefore, every $\mathrm{HN}$ factor $F$ of $E_{1}$ with respect to $\mathcal{P}$ has phase at most $\phi$. By (1), it follows that $W(F)$ has phase less than $\phi+\epsilon$, and thus the same holds for the phase $\psi^{+}(E)$ of $W\left(E_{1}\right)$.

A similar argument shows $\psi^{-}(E)>\phi(E)-\epsilon$, thus proving the claim.

Corollary 2.10 The map $\mathcal{Z}: \operatorname{Stab}_{\Lambda}(\mathcal{D}) \rightarrow \operatorname{Hom}(\Lambda, \mathbb{C})$, see Definition 2.1, is locally injective.

Moreover, consider a section $U \rightarrow \operatorname{Stab}_{\Lambda}(\mathcal{D}), Z \mapsto \sigma_{Z}=\left(Z, \mathcal{P}_{Z}\right)$ of $\mathcal{Z}$ defined on a subset $U \subset \operatorname{Hom}(\Lambda, \mathbb{C})$, such that every $\sigma_{Z}$ satisfies the support property with respect to $Q$. Assume that $U$ can be covered by open subsets $V_{i}$ such that for all $Z, Z^{\prime} \in V_{i}$ we have $d\left(\mathcal{P}_{Z}, \mathcal{P}_{Z^{\prime}}\right)<\frac{1}{4}$. Then this section is continuous.

Proof For the first statement, we just set $Z=W$ in the Lemma, to obtain $d(\mathcal{P}, \mathcal{Q})=0$.

For the second statement, we only need to show that (1) holds in a neighbourhood of $Z \in \operatorname{Hom}(\Lambda, \mathbb{C})$ when $Q$ is negative definite on $\operatorname{Ker} Z$. Choose any metric on $\Lambda_{\mathbb{R}}$; then clearly (1) only needs to be checked for vectors with unit length. Since $Q\left(\_\right) \geq 0$ defines a compact subset of the unit sphere, on which $|Z(v)|$ is a positive continuous function, the claim follows.

Morever, Proposition 2.3 gives a continuous action of $\widetilde{G L}_{2}^{+}(\mathbb{R})$ on $\operatorname{Stab}_{\Lambda}(\mathcal{D})$.

\section{Harder-Narasimhan filtrations via the Harder-Narasimhan polygon}

Throughout this section, let $\mathcal{A}$ be an abelian category with a stability function $Z$.

Definition 3.1 The Harder-Narasimhan polygon $\mathrm{HN}^{Z}(E)$ of an object $E \in \mathcal{A}$ is the convex hull of the central charges $Z(A)$ of all subobjects $A \subset E$ of $E$.

(The trivial subobjects $A=0$ or $A=E$ are included in the definition.) The idea to consider this convex set in the context of slope-stability goes back at least 40 years [21]. 


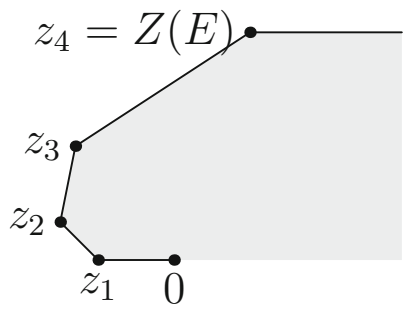

Fig. 1 Polyhedral on the left

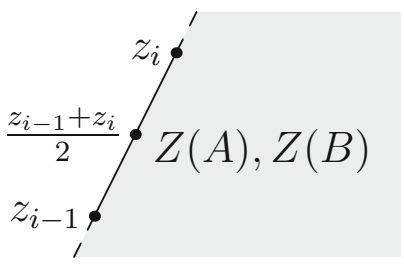

Fig. 2 Lemma 3.4

Definition 3.2 We say that the Harder-Narasimhan polygon $\mathrm{HN}^{Z}(E)$ of an object $E \in \mathcal{A}$ is polyhedral on the left if the set has finitely many extremal points $0=$ $z_{0}, z_{1}, \ldots, z_{m}=Z(E)$ such that $\mathrm{HN}^{Z}(E)$ lies to the right ${ }^{1}$ of the path $z_{0} z_{1} z_{2} \ldots z_{m}$; see Fig. 1.

In other words, the intersection of $\mathrm{HN}^{Z}(E)$ with the closed half-plane to the left of the line through 0 and $Z(E)$ is the polygon with vertices $z_{0}, z_{1}, \ldots, z_{m}$. Our proof of Theorem 1.2 is based on the following well-known statement; we provide a proof for completeness:

Proposition 3.3 The object $E$ has a Harder-Narasimhan filtration with respect to $Z$ if and only if its Harder-Narasimhan polygon $\mathrm{HN}^{Z}(E)$ is polyhedral on the left.

Assume that $\mathrm{HN}^{Z}(E)$ is polyhedral on the left. For each $i=1, \ldots, m$, choose a subobject $E_{i} \subset E$ such that $Z\left(E_{i}\right)=z_{i}$. (This exists as $z_{i}$ is extremal.)

Lemma 3.4 This is a filtration, i.e. $E_{i-1} \subset E_{i}$ for $i=1, \ldots, m$.

Proof Let $A:=E_{i-1} \cap E_{i} \subset E$ be the intersection of two subsequent objects, and $B:=E_{i-1}+E_{i} \subset E$ be their span inside $E$. Then there is a short exact sequence

$$
A \hookrightarrow E_{i-1} \oplus E_{i} \rightarrow B
$$

Hence the midpoint of $Z(A)$ and $Z(B)$ is also the midpoint of $z_{i-1}$ and $z_{i}$, see also Fig. 2.

On the other hand, $Z(A), Z(B)$ lie in $\mathrm{HN}^{Z}(E)$; by convexity and the choice of $z_{i-1}, z_{i}$, they both have to lie either in the open half-plane to the right of the line

\footnotetext{
$\overline{1 \text { By "to the right of" a given path } \gamma \text { we mean all points of the form } z}+x$ where $z \in \gamma$ and $x \in \mathbb{R}_{\geq 0}$.
} 


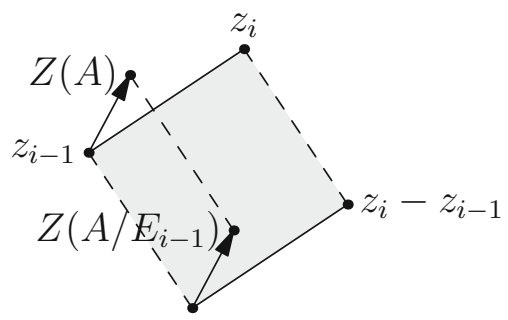

Fig. 3 Lemma 3.5

$\left(z_{i-1} z_{i}\right)$, or on the line segment $\overline{z_{i-1} z_{i}}$. The former would be a contradiction to the previous paragraph, and so $Z(A) \in \overline{z_{i-1} z_{i}}$.

Since $A \subset E_{i-1}$, this implies $Z(A)=z_{i-1}$ and $A \cong E_{i-1}$; therefore, $E_{i-1} \subset E_{i}$.

Lemma 3.5 The filtration quotient $E_{i} / E_{i-1}$ is semistable.

Proof Otherwise, there is an object $A$ with $E_{i-1} \subset A \subset E_{i}$ such that $A / E_{i-1}$ has bigger phase than $E_{i} / E_{i-1}$, see Fig. 3. It follows that $Z(A)$ lies to the left of the line segment $\overline{z_{i-1} z_{i}}$. Since $A \subset E$ and hence $Z(A) \in \mathrm{HN}^{Z}(E)$, this is a contradiction.

Proof of Proposition 3.3 The phase of $E_{i} / E_{i-1}$ is determined by the argument of $z_{i}-$ $z_{i-1}$; by convexity this shows $\phi\left(E_{1} / E_{0}\right)>\cdots>\phi\left(E_{m} / E_{m-1}\right)$, and so the $E_{i}$ form a HN filtration.

Conversely, assume that we are given a $\mathrm{HN}$ filtration $0=E_{0} \hookrightarrow E_{1} \hookrightarrow \cdots \hookrightarrow$ $E_{m}$ and a subobject $A \hookrightarrow E$. We have to show that $Z(A)$ lies to the right of the path $z_{0} z_{1} \ldots z_{m}$ with vertices $z_{i}:=Z\left(E_{i}\right)$. By induction on $m$, we may assume that $Z\left(A \cap E_{m-1}\right)$ lies to the right of the path $z_{0} z_{1} \ldots z_{m-1}$. On the other hand, $A /\left(A \cap E_{m-1}\right)$ is a subobject of $E_{m} / E_{m-1}$, which is semistable; thus its central charge $Z\left(A /\left(A \cap E_{m-1}\right)\right)$ lies to the right of the line segment from 0 to $z_{m}-z_{m-1}$. Therefore, $Z(A)=Z\left(A \cap E_{m-1}\right)+Z\left(A /\left(A \cap E_{m-1}\right)\right.$ lies to the right of the path $z_{0} z_{1} \ldots z_{m}$ as claimed.

Corollary 3.6 Given $E \in \mathcal{A}$, assume that there are only finitely many classes $v(A)$ of subobjects $A \subset E$ with $\Re Z(A)<\max \{0, \Re Z(E)\}$. Then $E$ admits a HN filtration.

\section{Linear algebra lemmas}

Throughout Sects. 4, 5 and 6, we fix a quadratic form $Q$ on $\Lambda_{\mathbb{R}}$ with:

Assumption 4.1 The quadratic form $Q$ has signature $(2, \operatorname{rk} \Lambda-2)$.

Lemma 4.2 Let $Z: \Lambda \rightarrow \mathbb{C}$ be a group homomorphism such that $Q$ is negative definite on $K:=\operatorname{Ker} Z \subset \Lambda_{\mathbb{R}}$. Let $\|\cdot\|$ be the norm on $K$ associated to $-\left.Q\right|_{K}$, and let 
$p: \Lambda_{\mathbb{R}} \rightarrow K$ be the orthogonal projection with respect to $Q$. After replacing $Z$ by $a$ $\mathrm{GL}_{2}^{+}(\mathbb{R})$-translate, we have

$$
Q(v)=|Z(v)|^{2}-\|p(v)\|^{2}
$$

Proof Let $K^{\perp}$ be the orthogonal complement of $K$. As $\left.Z\right|_{K^{\perp}}$ is injective, Assumption 4.1 can only hold if $Q$ is positive definite on $K^{\perp}$, and if $\left.Z\right|_{K^{\perp}}: K^{\perp} \rightarrow \mathbb{C}$ is an isomorphism. $\mathrm{Up}$ to the $\mathrm{GL}_{2}^{+}(\mathbb{R})$-action, we may assume this to be an isometry. Then the the claim follows.

Remark 4.3 In [10], this normalisation is used with the Mukai quadratic form replacing $Q$.

Consider the subset in $\operatorname{Hom}(\Lambda, \mathbb{C})$ of central charges whose kernel is negative definite with respect to $Q$; let $\mathcal{P}_{Z}(Q)$ be its connected component containing $Z$.

Lemma 4.4 Let $Q, Z$ and $K$ be as in Lemma 4.2.

1. For each $Z^{\prime} \in \mathcal{P}_{Z}(Q)$ there exists a unique $g \in \mathrm{GL}_{2}^{+}(\mathbb{R})$ and a linear map $u: K \rightarrow \mathbb{C}$ with $\|u\|<1$ such that

$$
g Z^{\prime}=Z+u \circ p
$$

2. Up to the action of $\mathrm{GL}_{2}^{+}(\mathbb{R})$, we can break up this deformation of $Z$ into a pure real and a purely imaginary part with analogous properties: there exist $u_{\mathbb{R}}, u_{i \mathbb{R}}$, $Z_{1}$ and $g_{1}, g_{2} \in \mathrm{GL}_{2}^{+}(\mathbb{R})$, depending continuously on $Z^{\prime}$, such that

(a) $u_{\mathbb{R}}: K \rightarrow \mathbb{R}$ and $u_{i \mathbb{R}}: \operatorname{Ker} Z_{1} \rightarrow \mathbb{R}$ satisfy $\left\|u_{\mathbb{R}}\right\|<1$, $\left\|u_{i \mathbb{R}}\right\|<1$,

(b) $Z_{1}:=Z+u_{\mathbb{R}} \circ p$,

(c) Eq. (2) holds with $Z$ and $p$ replaced by $g_{1} Z_{1}$ and $p_{1}: \Lambda_{\mathbb{R}} \rightarrow \operatorname{Ker} Z_{1}$, and

(d) $g_{2} Z^{\prime}=g_{1} Z_{1}+i u_{i \mathbb{R}} \circ p_{1}$, where $p_{1}: \Lambda_{\mathbb{R}} \rightarrow \operatorname{Ker} Z_{1}$ is the orthogonal projection.

Proof The restriction of $Z^{\prime}$ to the orthogonal complement $K^{\perp}$ is an isomorphism for all $Z^{\prime} \in \mathcal{P}_{Z}(Q)$. Hence there exists a unique $g \in \mathrm{GL}_{2}(\mathbb{R})$ such that $\left.g Z^{\prime}\right|_{K^{\perp}}=\left.Z\right|_{K^{\perp}}$; since $\mathcal{P}_{Z}(Q)$ is connected, in fact $g \in \mathrm{GL}_{2}^{+}(\mathbb{R})$. Let $u:=\left.g Z^{\prime}\right|_{\mathrm{Ker} Z}$; then $g Z^{\prime}-Z=$ $u \circ p$ holds both on $K$ and $K^{\perp}$, and thus on all of $\Lambda_{\mathbb{R}}$.

Next, we prove $\|u\|<1$. Otherwise, let $v \in K$ with $\|v\|=1$ and $|u(v)| \geq 1$, and let $\bar{v} \in K^{\perp}$ be such that $Z(\bar{v})=u(v)$. Then $Z^{\prime}(v-\bar{v})=0$, but $Q(v-\bar{v})=$ $|u(v)|^{2}-\|v\|^{2} \geq 0$ in contradiction to $Q$ being negative definite on $\operatorname{Ker} Z^{\prime}$. This completes the proof of 1 .

Now let $u_{\mathbb{R}}:=\Re u$, and let $Z_{1}$ be as above. If we write $\Lambda_{\mathbb{R}}=K \oplus K^{\perp}$ and identify $K^{\perp}$ with $\mathbb{C}$ via $Z$, then $\operatorname{Ker} Z_{1}$ is the graph of $-u_{\mathbb{R}}$, and its orthogonal complement is the graph of $\mathbb{C} \rightarrow K, z \mapsto \Re z \cdot u^{\vee}$, where $u^{\vee}$ corresponds to $u$ under the identification $K \cong K^{\vee}$ induced by the symmetric form associated to $\|\cdot\|$. A straightforward computation shows that $g_{1} \circ Z_{1}$ induces an isometry $\left(\operatorname{Ker} Z_{1}\right)^{\perp} \rightarrow \mathbb{C}$ for $g_{1}:=\left(\begin{array}{cc}\left(1+\left\|u_{\mathbb{R}}\right\|^{2}\right)^{-\frac{1}{2}} & 0 \\ 0 & 1\end{array}\right) \in \mathrm{GL}_{2}^{+}(\mathbb{R}) ;$ by the proof of Lemma 4.2 this implies (c). 
Moreover, $g_{1} g Z^{\prime}-g_{1} Z_{1}=g_{1} \circ \Im u \circ p$ is completely imaginary. Applying part 1 . to $g_{1} g Z^{\prime}$ and $g_{1} Z_{1}$ then shows part (d).

\section{Real variations of the central charge}

The key lemma, proved in this section and the next, treats the case where only the real part of the central charge is varying:

Lemma 5.1 Consider a stability condition $\sigma=(Z, \mathcal{P})$, satisfying the support property with respect to $Q$, and assume that (2) holds with $p$ and $\|\cdot\|$ as defined in Lemma 4.2. Given $u: \operatorname{Ker} Z \rightarrow \mathbb{R}$ with $\|u\|<1$, there is a stability condition $\tau=(W, \mathcal{Q})$ with $W=Z+u \circ p$, satisfying the support property with respect to $Q$, and with $d(\mathcal{P}, \mathcal{Q})<\frac{\|u\|}{2}$.

Proof of Theorem 1.2, assuming Lemma 5.1 and Assumption 4.1 Lemma 5.1 automatically also applies to purely imaginary variations of the central charge as in Lemma 4.4.(d), due to the $\widetilde{G L}_{2}^{+}(\mathbb{R})$-action. We may assume that $Q$ and the central charge satisfy (2); then Lemmas 4.4 and 5.1 combined with the $\widetilde{G L}_{2}^{+}(\mathbb{R})$-action give a set-theoretic section $\mathcal{P}_{Z}(Q) \rightarrow \operatorname{Stab}_{\Lambda}(\mathcal{D})$.

Since $\mathrm{GL}_{2}^{+}(\mathbb{R})$ acts continuously on the compact set $S^{1}$, there exists an open neighbourhood $B_{\epsilon}(1)$ of $1 \in \widetilde{G L}_{2}^{+}(\mathbb{R})$ such that $|\tilde{g} . \phi-\phi|<\epsilon$ for all $\tilde{g} \in B_{\epsilon}(1)$. It follows that if $(\mathcal{Q}, W)=\tilde{g} .(\mathcal{P}, Z)$, then $d(\mathcal{Q}, \mathcal{P})<\epsilon$. Then there is an open neighborhood $U_{\epsilon}(Z)$ of $Z$ in $\mathcal{P}_{Z}(Q)$ where, in the notation of Lemma $4.4,\left\|u_{\mathbb{R}}\right\|<\epsilon,\left\|u_{i \mathbb{R}}\right\|<\epsilon$, and $g_{1}, g_{2}$ lift to $\tilde{g}_{1}, \tilde{g}_{2} \in B_{\epsilon}(1)$. Then our lift $\tau=(Z, \mathcal{Q})$ of any $Z^{\prime}$ in this open neighbourhood satisfies $d(\mathcal{Q}, \mathcal{P})<\frac{\epsilon}{2}+\epsilon+\frac{\epsilon}{2}+\epsilon$.

By Corollary 2.10, our section is continuous on $U_{\epsilon}(Z)$ for $\epsilon \leq \frac{1}{12}$. Since the neighbourhoods $U_{\frac{1}{12}}\left(Z^{\prime}\right)$ for $Z^{\prime} \in \mathcal{P}_{Z}(\mathcal{Q})$ cover $\mathcal{P}_{Z}(\mathcal{Q})$, and since the lifts constructed on each such neighbourhood agree on the overlaps by the local injectivity of $\mathcal{Z}$, this proves the Theorem.

Lemma 5.1 is simpler to prove since it allows (and forces) us to leave the heart $\mathcal{A}:=$ $\mathcal{P}(0,1]$ unchanged: we will apply Proposition 2.6 and prove that $(\mathcal{A}, W)$ produces a stability condition.

Lemma 5.2 In the situation of Lemma 5.1, $W=Z+u \circ p$ is a stability function on $\mathcal{A}$.

Proof Consider $E \in \mathcal{A}$; if $\Im Z(E)=\Im W(E)>0$, there is nothing to prove. Otherwise, $E$ is semistable with $Z(E) \in \mathbb{R}_{<0}$ and thus $\|p(E)\| \leq-Z(E)$. From $\|u\|<1$ we conclude

$$
W(E)=Z(E)+u \circ p(E) \leq Z(E)+\|u\|\|p(E)\|<Z(E)-Z(E)=0 .
$$

We will use Proposition 3.3 and Corollary 3.6 to prove that $(\mathcal{A}, W)$ satisfies the HN property. 


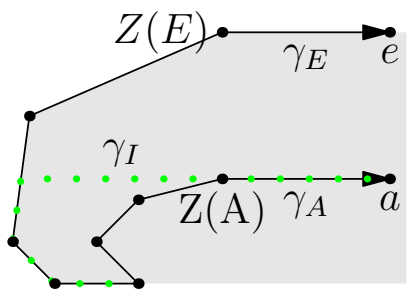

Fig. 4 Proof of Lemma 5.5

Let us define the mass $m^{Z}(E)$ of $E \in \mathcal{A}$ with respect to $Z$ as the length of the boundary of $\mathrm{HN}^{Z}(E)$ on the left between 0 and $Z(E)$.

Lemma 5.3 For all $E \in \mathcal{A}$ we have $\|p(E)\| \leq m^{Z}(E)$.

Proof If $E$ is semistable, then $0 \leq Q(E)=|Z(E)|^{2}-\|p(E)\|^{2}=\left(m^{Z}(E)\right)^{2}-$ $\|p(E)\|^{2}$, which is exactly the claim. Otherwise, consider the HN filtration $E_{0} \hookrightarrow$ $E_{1} \hookrightarrow \ldots \hookrightarrow E_{m}=E$. Combined with the triangle inequality, this gives

$$
\begin{aligned}
\|p(E)\| & \leq \sum_{i}\left\|p\left(E_{i} / E_{i-1}\right)\right\| \leq \sum_{i}\left|Z\left(E_{i} / E_{i-1}\right)\right| \\
& =\sum_{i}\left|Z\left(E_{i}\right)-Z\left(E_{i-1}\right)\right|=m^{Z}(E) .
\end{aligned}
$$

The following Lemma needs no proof:

Lemma 5.4 If $A \subset E$, then $\mathrm{HN}^{Z}(A) \subset \mathrm{HN}^{Z}(E)$.

Lemma 5.5 Given any subobject $A \subset E$, we have

$$
m^{Z}(A)-\Re Z(A) \leq m^{Z}(E)-\Re Z(E) .
$$

Proof This follows from the previous Lemma, convexity and a picture, see Fig. 4. Indeed, choose $x>\Re Z(A), \mathfrak{R} Z(E)$; let $a=x+i \Im Z(A)$ and $e=x+i \Im Z(E)$. Let $\gamma_{A}$ be the path that follows the boundary of $\mathrm{HN}^{Z}(A)$ from 0 to $Z(A)$, and then continues horizontally to $a$; similarly $\gamma_{E}$ follows the boundary of $\operatorname{HN}^{Z}(E)$ and then continues to $e$. Their lengths are given as

$$
\left|\gamma_{A}\right|=m^{Z}(A)+x-\mathfrak{R} Z(A), \quad\left|\gamma_{E}\right|=m^{Z}(E)+x-\mathfrak{R} Z(E) .
$$

On the other hand, convexity and Lemma 5.4 imply $\left|\gamma_{A}\right| \leq\left|\gamma_{E}\right|$; for example, if $\gamma_{I}$ denotes the intermediate path that follows the boundary of $\mathrm{HN}^{Z}(E)$ up to height $\Im Z(A)$ and then goes horizontally to $a$, we clearly have $\left|\gamma_{A}\right| \leq\left|\gamma_{I}\right| \leq\left|\gamma_{E}\right|$. 
Lemma 5.6 Given $C \in \mathbb{R}$, the set of $v(A) \in \Lambda$ for subobjects $A \subset E$ with $\Re W(A)<$ $C$ is finite.

Proof Given any such $A$, we use Lemmas 5.3 and 5.5 to obtain

$$
\begin{aligned}
C & >\mathfrak{R} W(A) \geq \mathfrak{R}(A)-\|u\|\|p(A)\| \\
& >(1-\|u\|) \Re Z(A)-\|u\|\left(m^{Z}(A)-\Re Z(A)\right) \\
& \geq(1-\|u\|) \Re Z(A)-\|u\|\left(m^{Z}(E)-\mathfrak{R} Z(E)\right) .
\end{aligned}
$$

Since $\|u\|<1$, this bounds $\Re Z(A)$ from above. On the other hand, $Z(A) \in \operatorname{HN}^{Z}(E)$, and thus $Z(A)$ is constrained to lie in a compact region of $\mathbb{C}$. Using Lemmas 5.5 and 5.3 again, this gives an upper bound first for $m^{Z}(A)$ and consequently for $\|p(A)\|$. Hence $v(A)$ is contained in a compact region of $\Lambda \otimes \mathbb{R}$ depending only on $E$ and $C$, and the claim follows.

Lemma 5.6 and Corollary 3.6 imply the existence of $\mathrm{HN}$ filtrations for $W$ on $\mathcal{A}$, and thus yield a pre-stability condition $\tau=(\mathcal{A}, W)$ by Proposition 2.6; write $\mathcal{Q}$ for the associated slicing.

Lemma 5.7 The pre-stability condition $\tau=(W, \mathcal{Q})$ satisfies $d(\mathcal{P}, \mathcal{Q})<\frac{\|u\|}{2}$.

Proof We apply Lemma 2.9: by construction, by (2), and by the assumption $\|u\|<1$ we have

$$
\begin{aligned}
& \frac{|W(v)-Z(v)|}{|Z(v)|} \\
& =\frac{|u \circ p(v)|}{|Z(v)|} \leq\|u\| \frac{\|p(v)\|}{|Z(v)|} \leq\|u\|<\sin \pi \frac{\|u\|}{2} \text { for } v \in \Lambda \text { with } Q(v) \geq 0 .
\end{aligned}
$$

\section{The support property is preserved}

It remains to show that $(\mathcal{A}, W)$ satisfies the support property with respect to $Q$, i.e. $Q(v(E)) \geq 0$ for all $W$-stable $E \in \mathcal{A}$. The basic reason is that this inequality preserved by wall-crossing:

Lemma 6.1 Let $\sigma=(Z, \mathcal{P})$ be pre-stability condition. Assume that $Q$ is a nondegenerate quadratic form on $\Lambda_{\mathbb{R}}$ of signature $(2, \operatorname{rk} \Lambda-2)$ such that $Q$ is negative definite on $\operatorname{Ker} Z$. If $E$ is strictly $\sigma$-semistable and admits a Jordan-Hölder filtration with factors $E_{1}, \ldots, E_{m}$, and if $Q\left(v\left(E_{i}\right)\right) \geq 0$ for $i=1, \ldots, m$, then $Q(v(E)) \geq 0$.

Proof We apply Lemma 4.2; then $Q(v) \geq 0$ is equivalent to $|Z(v)| \geq\|p(v)\|$. We obtain

$$
|Z(E)|=\sum_{i}\left|Z\left(E_{i}\right)\right| \geq \sum_{i}\left\|p\left(v\left(E_{i}\right)\right)\right\| \geq\left\|\sum_{i} p\left(v\left(E_{i}\right)\right)\right\|=\|p(v(E))\|
$$


where the first equality holds since the central charges of all $E_{i}$ are aligned, the first inequality holds by assumption, and the second inequality is the triangle inequality.

The proof strategy is thus clear: we use wall-crossing for the path of stability functions $Z_{t}=Z+t \cdot u \circ p$ on $\mathcal{A}$, for $0 \leq t \leq 1$. If $E \in \mathcal{A}$ is $Z_{1}$-stable with $Q(v(E))<0$, then it must be $Z_{0}$-unstable; wall-crossing gives a $t \in[0,1)$ such that $E$ is strictly $Z_{t}$-semistable; by the Lemma, one of its Jordan-Hölder factors will also violate the inequality, and we proceed by induction. To conclude, we have to show that we can find such a wall, and that this process terminates.

Lemma 6.2 Given two objects $A, E \in \mathcal{A}$, denote their phases with respect to $Z_{t}$ by $\phi^{t}(A), \phi^{t}(E)$, respectively. If the set of $t \in[0,1]$ with $\phi^{t}(A) \geq \phi^{t}(E)$ is non-empty, then it is a closed subinterval of $[0,1]$ containing one of its endpoints.

Proof The condition is equivalent to $\frac{-\Re Z_{t}(A)}{\Im Z_{t}(A)} \geq \frac{-\Re Z_{t}(E)}{\Im Z_{t}(E)}$, which is a linear inequality in $t$.

Consider the polygon whose vertices are the extremal points of $\mathrm{HN}^{Z_{0}}(E)$ on the left; we will call this the truncated $H N$ polygon of $E$, see Fig. 5. Note that if $A \subset E$ is a subobject with $\phi^{0}(A) \geq \phi^{0}(E)$, then $Z_{0}(A)$ is contained in the truncated $\mathrm{HN}$ polygon of $E$; by Lemmas 5.5 and 5.3 there are only finitely many classes $v(A)$ of such subobjects.

Lemma 6.3 Every $Z_{1}$-semistable object $E \in \mathcal{A}$ satisfies $Q(E) \geq 0$.

Proof Otherwise, $E$ must be $Z_{0}$-unstable. By Lemma 6.2 and the following observation, there are only finitely many classes $v(A)$ of subobjects $A \hookrightarrow E$ that destabilise $E$ with respect $Z_{t}$ for any $t \in[0,1]$. Hence there is a wall $t_{1} \in(0,1]$ such that $E$ is strictly semistable with respect to $Z_{t_{1}}$, and moreover $E$ admits a Jordan-Hölder filtration with respect to $Z_{t_{1}}$. By Lemma 6.1 , there are subobjects $G_{1} \hookrightarrow F_{1} \hookrightarrow E$ of the same phase, such that $F_{1} / G_{1}$ is $Z_{t_{1}}$-stable with $Q\left(v\left(F_{1} / G_{1}\right)\right)<0$.

Applying the same logic to $F_{1} / G_{1}$, we obtain $t_{2} \in\left(0, t_{1}\right)$ and subobjects $G_{1} \subset$ $G_{2} \subset F_{2} \subset F_{1} \subset E$ such that $F_{2} / G_{1}, G_{2} / G_{1}$ and $F_{1} / G_{1}$ all have the same phase with respect to $t_{2}$, and such that $Q\left(v\left(F_{2} / G_{2}\right)\right)<0$. Continuing by induction, we obtain a sequence $t_{1}>t_{2}>t_{3}>\ldots$ in $(0,1)$ and chains of subobjects $G_{1} \subset G_{2} \subset$ $G_{3} \subset \cdots \subset E$ and $E \supset F_{1} \supset F_{2} \supset F_{3} \supset \ldots$

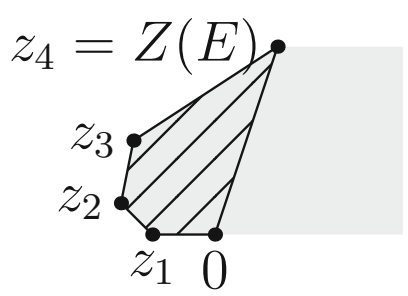

Fig. 5 The truncated $\mathrm{HN}$ polygon 
Lemma 6.2 gives $\phi^{t_{2}}\left(F_{1}\right) \geq \phi^{t_{2}}(E)$ and $\phi^{t_{2}}\left(G_{1}\right) \geq \phi^{t_{2}}(E)$. Since $Z_{t_{2}}\left(F_{2}\right)$ lies on the line segment connecting $Z_{t_{2}}\left(F_{1}\right)$ and $Z_{t_{2}}\left(G_{1}\right)$, we also have $\phi^{t_{2}}\left(F_{2}\right) \geq \phi^{t_{2}}(E)$ (and therefore $\phi^{t}\left(F_{2}\right) \geq \phi^{t}(E)$ for all $t \in\left[0, t_{2}\right]$ ); similarly for $G_{2}$. Continuing by induction, we conclude $\phi^{0}\left(F_{i}\right) \geq \phi^{0}(E)$. This is a contradiction to the observation above that there are only finitely many classes $v(A)$ of subobjects $A \subset E$ with $Z_{0}(A)$ lying in the truncated $\mathrm{HN}$ polygon.

This concludes the proof of Lemma 5.1, and thus of Theorem 1.2 under the Assumption 4.1.

\section{Reductions}

Finally, we will show that we can always reduce the situation to the case where Assumption 4.1 holds. By abuse of language, we call a quadratic form degenerate or non-degenerate if the associated symmetric bilinear form is degenerate or nondegenerate, respectively.

Lemma 7.1 Assume that the quadratic form $Q$ on $\Lambda_{\mathbb{R}}$ is degenerate. Then there exists an injective map $\Lambda_{\mathbb{R}} \hookrightarrow \bar{\Lambda}$ of real vector spaces and a non-degenerate quadratic form $\bar{Q}$ on $\bar{\Lambda}$, extending $Q$, such that any central charge $Z: \Lambda_{\mathbb{R}} \rightarrow \mathbb{C}$ with kernel negative definite with respect to $Q$ extends to a central charge $\bar{Z}: \bar{\Lambda} \rightarrow \mathbb{C}$ with kernel negative definite with respect to $\bar{Q}$.

Proof Let $N \hookrightarrow \Lambda_{\mathbb{R}}$ be the null space of $Q$; we will only treat the case $\operatorname{dim}_{\mathbb{R}} N=1$ (otherwise, we can iterate the construction that follows). Choose a splitting $\Lambda_{\mathbb{R}} \cong$ $N \oplus C$; then for $n \in N, c \in C$, we have $Q(n \oplus c)=Q(c)$. Let $\overline{\Lambda_{\mathbb{R}}}:=N \oplus N^{\vee} \oplus C$, let $q$ be the canonical quadratic form on the hyperbolic plane $N \oplus N^{\vee}$, and set $\bar{Q}:=\left.q \oplus Q\right|_{C}$.

Given $Z$ as above, the restriction $\left.Z\right|_{N}$ is injective, and we may assume that $Z$ maps $N$ to the real line. Let $n \in N$ be such that $Z(n)=1$, and let $n^{\vee} \in N^{\vee}$ be the dual vector with $\left(n, n^{\vee}\right)=1$. We claim that for $\alpha \gg 0$, the extension of $Z$ defined by $Z^{\prime}\left(n^{\vee}\right)=\alpha$ has the desired property.

Let $K:=\operatorname{Ker} Z$; then the kernel of $Z^{\prime}$ is contained in $N \oplus N^{\vee} \oplus K$, and given by vectors of the form $a \cdot n-\frac{a}{\alpha} \cdot n^{\vee}+k$ for $k \in K, a \in \mathbb{R}$. For such vectors, we have

$$
Q\left(a \cdot n-\frac{a}{\alpha} \cdot n^{\vee}+k\right)=-\frac{2 a^{2}}{\alpha}-\frac{2 a}{\alpha}\left(n^{\vee}, k\right)+Q(k) .
$$

This is a quadratic function in $a$ with negative constant term; its discriminant is negative if

$$
\alpha>\max \left\{\frac{\left(n^{\vee}, k\right)^{2}}{-Q(k)}: k \in K, k \neq 0\right\}
$$

(which is finite since $-Q(\cdot)$ is a positive definite form on $K$ ).

Replacing $\Lambda$ by $\Lambda \oplus \mathbb{Z}$ and $v$ by

$$
K(\mathcal{D}) \stackrel{v}{\rightarrow} \Lambda \hookrightarrow \Lambda \oplus \mathbb{Z}
$$


we can therefore restrict to the case where $Q$ is non-degenerate: given a path $Z_{t}$ of central charges in $\operatorname{Hom}\left(\Lambda_{\mathbb{R}}, \mathbb{C}\right)$ that are negative definite with respect to $Q$, we can choose extensions $\bar{Z}_{t}$ as in the Lemma that form a continuous path in $\operatorname{Hom}(\bar{\Lambda}, \mathbb{C})$. If we can lift the latter path to a path of stability conditions $\bar{\sigma}_{t}=\left(\bar{Z}_{t}, \mathcal{P}_{t}\right)$ that satisfy the support property with respect to $\bar{Q}$, then $\sigma_{t}:=\left(Z_{t}, \mathcal{P}_{t}\right)$ is a path of stability conditions satisfying the support property with respect to $Q$. The reduction to the case where $Q$ has signature $(2, \mathrm{rk} \Lambda-2)$ works similarly:

Lemma 7.2 Assume that $Q$ is non-degenerate and of signature $(p, \operatorname{rk} \Lambda-p)$ for $p \in$ $\{0,1\}$. Let $\bar{\Lambda}:=\Lambda_{\mathbb{R}} \oplus \mathbb{R}$, and let $\bar{Q}$ be the extension given by $\bar{Q}(v, \alpha)=Q(v)+\alpha^{2}$ for $v \in \Lambda_{\mathbb{R}}$ and $\alpha \in \mathbb{R}$. Then any central charge $Z$ on $\Lambda_{\mathbb{R}}$ whose kernel is negative definite with respect to $Q$ extends to a central charge $\bar{Z}$ on $\bar{\Lambda}$ whose kernel is negative definite with respect to $\bar{Q}$.

Proof We claim that there exists $z \in \mathbb{C}$ such that for all $v \in \Lambda_{\mathbb{R}}$ with $Z(v)=z$, we have $Q(v)<-1$. Indeed, let $K \subset \Lambda_{\mathbb{R}}$ be the kernel of $Z$, and let $K^{\perp}$ be its orthogonal complement. Then clearly we may assume $v \in K^{\perp}$. Since the restriction of $Z$ to $K^{\perp}$ is injective, and since $K^{\perp}$ either has rank one, or has signature $(1,-1)$ with respect to $Q$, the claim is evident.

Using the claim, we can set $\bar{Z}(v, \alpha):=Z(v)+\alpha z$.

This concludes the proof of Theorem 1.2.

\section{Application}

Proof of Corollary 1.3 Using the same arguments as in the previous section, we may assume that the Mukai pairing on $\Lambda$ has signature $(2, \operatorname{rk} \Lambda-2)$.

By Serre duality, any $\sigma$-stable object $E \in \mathcal{D}$ satisfies $\operatorname{Hom}(E, E[i])=0$ for $i<0$ or $i>3$ and $\operatorname{Hom}(E, E)=\mathbb{C}=\operatorname{Hom}(E, E[2])$; therefore, $(v(E), v(E)) \geq-2$. Moreover, Serre duality induces a non-degenerate symplectic form on $\operatorname{Ext}^{1}(E, E)$, and it has even dimension; thus $v(E)$ is a root, or $(v(E), v(E)) \geq 0$.

Let $\sigma=(Z, \mathcal{P})$ be a stability condition with $Z \in \mathcal{P}_{0}(\mathcal{D})$. As in Lemma 4.2 we may assume

$$
(v, v)=|Z(v)|^{2}-\|p(v)\|^{2},
$$

where $p: \Lambda_{\mathbb{R}} \rightarrow \operatorname{Ker} Z$ is the orthogonal projection onto the kernel of $Z$, and where $\|\cdot\|$ denotes the norm on $\operatorname{Ker} Z$ induced by the negative of the Mukai pairing. We claim that

$$
C:=\inf \{|Z(\delta)|: \delta \in \Lambda,(\delta, \delta)=-2\}>0 .
$$

Indeed, if $|Z(\delta)| \leq 1$, then $\|p(\delta)\| \leq \sqrt{3}$; as $|Z(\cdot)|+\|p(\cdot)\|$ is a norm on $\Lambda_{\mathbb{R}}$, there are only finitely many integral classes satisfying both inequalities. Since $Z(\delta) \neq 0$ by assumption, the claim follows. 
Now set

$$
Q(v):=(v, v)+\frac{2}{C^{2}}|Z(v)|^{2} .
$$

Clearly $Q$ is negative definite on $\operatorname{Ker} Z$ and depends only on $Z$. Moreover, $Q(\delta) \geq 0$ for all roots $\delta$, and $Q(v) \geq 0$ for all classes with $(v, v) \geq 0$; therefore, any stability condition $\sigma^{\prime}=\left(Z^{\prime}, \mathcal{P}^{\prime}\right)$ with $Z^{\prime} \in \mathcal{P}_{Z}(Q)$ satisfies the support property with respect to $Q$.

Theorem 1.2 shows that the restriction of $\mathcal{Z}$ to the preimage of $\mathcal{P}_{Z}(Q)$ is a covering of $\mathcal{P}_{Z}(Q)$. Since the neighbourhoods $\mathcal{P}_{Z}(Q)$ cover $\mathcal{P}_{0}(\mathcal{D})$, this completes the proof.

Acknowledgements I would like to thank Emanuele Macrì and Paolo Stellari; as indicated above, Theorem 1.2 first appeared with a different proof in our joint work [7]. I presented similar arguments in my lectures at the Hausdorff school on derived categories in Bonn, April 2016; I am grateful to the organisers for the opportunity, and the participants for their feedback. I would also like to thank Martin Gulbrandsen and François Charles for pointing out inaccuracies in the first arXiv version of this article, and the anonymous referee for detailed comments improving the exposition. My work was supported by the ERC-StG WallXBirGeom 337039.

Open Access This article is distributed under the terms of the Creative Commons Attribution 4.0 International License (http://creativecommons.org/licenses/by/4.0/), which permits unrestricted use, distribution, and reproduction in any medium, provided you give appropriate credit to the original author(s) and the source, provide a link to the Creative Commons license, and indicate if changes were made.

\section{References}

1. Arcara, D., Bertram, A., Coskun, I., Huizenga, J.: The minimal model program for the Hilbert scheme of points on $\mathbb{P}^{2}$ and Bridgeland stability. Adv. Math. 235, 580-626 (2013). arXiv:1203.0316

2. Anno, R., Bezrukavnikov, R., Mirković, I.: Stability conditions for Slodowy slices and real variations of stability. Mosc. Math. J. 15(2), 187-203, 403 (2015). arXiv:1108.1563

3. Bayer, A.: Wall-crossing implies Brill-Noether. Applications of stability conditions on surfaces. In Algebraic geometry: Salt Lake City 2015. Proc. Symp. Pure Math., vol. 97, pp. 3-27 (2018). arXiv: 1604.08261

4. Bayer, A., Bridgeland, T.: Derived automorphism groups of K3 surfaces of Picard rank 1. Duke Math. J. 166(1), 75-124 (2017). arXiv:1310.8266

5. Bayer, A., Macrì, E.: The space of stability conditions on the local projective plane. Duke Math. J. 160(2), 263-322 (2011). arXiv:0912.0043

6. Bayer, A., Macrì, E.: MMP for moduli of sheaves on K3s via wall-crossing: nef and movable cones. Lagrangian fibrations. Invent. Math. 198(3), 505-590 (2014). arXiv:1301.6968

7. Bayer, A., Macrì, E., Stellari, P.: The space of stability conditions on abelian threefolds, and on some Calabi-Yau threefolds. Invent. math., pp. 1-65 (2016), arXiv:1410.1585

8. Bayer, A., Macrì, E., Toda, Y.: Bridgeland stability conditions on threefolds I: Bogomolov-Gieseker type inequalities. J. Algebra. Geom. 23(1), 117-163 (2014). arXiv:1103.5010

9. Bridgeland, T.: Stability conditions on triangulated categories. Ann. Math. (2) 166(2), 317-345 (2007). arXiv:math/0212237

10. Bridgeland, T.: Stability conditions on $K 3$ surfaces. Duke Math. J. 141(2), 241-291 (2008). arXiv:math/0307164

11. Bridgeland, T.: Spaces of stability conditions. In: Algebraic geometry-Seattle 2005. Part 1, volume 80 of Proc. Sympos. Pure Math., pp. 1-21. Amer. Math. Soc., Providence, RI (2009), arXiv:math.AG/0611510 
12. Bridgeland, Tom: Stability conditions and Kleinian singularities. Int. Math. Res. Not. IMRN 21(21), 4142-4157 (2009). arXiv:math/0508257

13. Bridgeland, T., Smith, I.: Quadratic differentials as stability conditions. Publ. Math. Inst. Hautes Études Sci. 121, 155-278 (2015). arXiv:1302.7030

14. Gaiotto, D., Moore, G.W., Neitzke, A.: Wall-crossing, Hitchin systems, and the WKB approximation. Adv. Math. 234, 239-403 (2013). arXiv:0907.3987

15. Huybrechts, D.: Introduction to stability conditions. In: Moduli spaces, volume 411 of London Math. Soc. Lecture Note Ser., pp. 179-229. Cambridge Univ. Press, Cambridge (2014). arXiv:1111.1745

16. Ikeda, A.: Stability conditions for preprojective algebras and root systems of Kac-Moody Lie algebras (2014). arXiv:1402.1392

17. Kontsevich, M., Soibelman, Y.: Stability structures, motivic Donaldson-Thomas invariants and cluster transformations (2008). arXiv:0811.2435

18. Li, C., Zhao, X.: Birational models of moduli spaces of coherent sheaves on the projective plane. Geom. Topol. 23(1), 347-426 (2019). arXiv:1603.05035

19. Meinhardt, S., Partsch, H.: Quotient categories, stability conditions, and birational geometry. Geom. Dedicata 173, 365-392 (2014). arXiv:0805.0492

20. Macrì, E., Schmidt, B.: Lectures on Bridgeland stability. In: Moduli of curves, vol. 21. Lect. Notes Unione Mat. Ital., pp. 139-211. Springer, Cham (2017). arXiv:1607.01262

21. Shatz, S.S.: Degeneration and specialization in algebraic families of vector bundles. Bull. Am. Math. Soc. 82(4), 560-562 (1976)

22. Thomas, R.P.: Stability conditions and the braid group. In: Superstring theory, volume 1 of Adv. Lect. Math. (ALM), pp. 209-233. Int. Press, Somerville, MA (2008). arXiv:math/0212214

23. Toda, Y.: Derived category of coherent sheaves and counting invariants In: Proceedings of the International Congress of Mathematicians-Seoul, vol. II. pp. 745-767 (2014). arXiv:1404.3814

Publisher's Note Springer Nature remains neutral with regard to jurisdictional claims in published maps and institutional affiliations. 